\title{
EFEITO DE PACLOBUTRAZOL COMO REGULADOR DE CRESCIMENTO E PRODUÇÃO DE FLORES DE GIRASSOL EM CULTIVO HIDROPÔNICO
}

\author{
Effect of paclobutrazol as regulator of growth in production of flowers \\ of sunflower in cultivo hidropônico
}

\author{
Christina da Silva Wanderley ${ }^{1}$, Roberto Rezende ${ }^{2}$, Carlos Alberto Bastos Andrade ${ }^{3}$
}

\begin{abstract}
RESUMO
Objetivou-se neste trabalho estudar os efeitos de um regulador de crescimento (paclobutrazol) sobre o crescimento e a produção de flores de girassol em vasos, crescidos sob sistema hidropônico. Foram utilizados dois genótipos de girassol (BRS Oásis e Helio 358), combinados com seis doses de paclobutrazol $\left(0 ; 0,5 ; 1 ; 2 ; 4\right.$ e 6 mg. $\left.\mathrm{L}^{-1}\right)$ e substrato inerte de sílica moída. A solução nutritiva foi a de Hoagland modificada (EPSTEIN \& BLOOM, 2004) e as plantas foram mantidas sob aeração constante. Para avaliar os efeitos do paclobutrazol sobre o crescimento das plantas (altura final e diâmetro de capítulo), as variáveis foram submetidas à análise de variação (teste F), utilizando-se rotinas do sistema SAS/STAT , aplicando-se, para os valores significativos a 5\% de probabilidade e a regressão linear e não linear para a análise dos parâmetros aferidos, em função das doses do regulador de crescimento. A análise dos dados permitiu concluir que há efeito do paclobutrazol sobre o crescimento e sobre a produção de flores de girassol, reduzindo a altura final das plantas e o diâmetro dos capítulos, sendo que doses elevadas causaram deformações nas plantas e má formação nas flores. Helio 358 apresentou-se mais adequado para a produção ornamental, sendo melhor dose entre 1 e $2 \mathrm{mgL}^{-1} \mathrm{de}$ paclobutrazol.
\end{abstract}

Termos para indexação: Paclobutrazol, diâmetro de capítulo, altura de planta, Helianthus annuus.

\begin{abstract}
This work had the aim of studying the effects of a growth regulator (paclobutrazol) on the growth and the production of sunflowers in vases, with hidroponic solution. Two sunflower genotipes were used (BRS Oásis and Helio 358), combined with six paclobutrazol doses $\left(0 ; 0,5 ; 1 ; 2 ; 4\right.$ and 6 mg.. $\left.\mathrm{L}^{-1}\right)$ and inert substratum of silex ground. The nutritious solution was a Hoagland modified (EPSTEIN \& BLOOM, 2004) and the plants were maintained under constant aeration. To evaluate the effects of the paclobutrazol on the growth of the plants (final height and head diameter), the variables were submitted to the variation analysis (F tests), being used routines of the system SAS/STAT, being applied, for the significant values at 5\% of probability and the lineal and not lineal regression for the analysis of the parameters checked, in function of the growth regulator doses. The analysis of the data allowed to conclude that there is effect of the paclobutrazol on the growth and on the production of sunflowers, reducing the final height of the plants and head diameter, and the high doses caused deformations in the plants and bad formation in the flowers. Helio 358 presented to be more adequate for the ornamental production, being the best dose between 1 and $2 \mathrm{mgL}^{-1} \mathrm{de}$ paclobutrazol.
\end{abstract}

Index terms: Paclobutrazol, head diameter, plant height, Helianthus annuus.

(Recebido em 20 de novembro de 2006 e aprovado em 23 de abril de 2007)

\section{INTRODUÇÃO}

O girassol (Helianthus annuus L.) é uma planta originária da América do Norte, que foi utilizada como planta ornamental e como hortaliça até o século XVIII, quando começou o seu uso como cultura comercial. Tem sido usada também como planta forrageira para alimentação animal, para alimentação de aves e como planta melífera e ornamental, além da produção de óleo para alimentação humana (DALL'AGNOL et al., 2005). Seu ciclo vegetativo depende de cada variedade ou híbrido, podendo ser de 90 até 150 dias no total. Segundo Reyes et al. (1985), o girassol pode ser plantado durante o ano todo e se adapta bem a condições variáveis de temperatura. Seu porte final depende do genótipo, mas normalmente ultrapassa 1,60 m, podendo chegar a $3,00 \mathrm{~m}$ de altura.

Esse grande crescimento natural tem sido entrave para que se explore o girassol como planta ornamental, já que apresenta facilidade de propagação, curto tempo para a colheita, e, segundo Anefalos \& Guilhoto (2003), uma inflorescência bastante atrativa e muito procurada para a confecção de arranjos e vasos.

\footnotetext{
'Engenheira Agrônoma, Mestre - Universidade Estadual de Maringá/UEM - Rua Deputado Fernando Ferrari, 141 - $86062-030$ - Londrina, PR chriscsw@sercomtel.com.br

${ }^{2}$ Engenheiro Agrônomo, Doutor, Professor - Departamento de Agronomia/PGA - Universidade Estadual de Maringá/UEM - Avenida Colombo, 5790, Bloco 32, sala 07 - 87020-900 - Maringá, PR - rrezende@uem.br

${ }^{3}$ Engenheiro Agrônomo, Doutor, Professor - Departamento de Agronomia/PGA - Universidade Estadual de Maringá/UEM - Avenida Colombo, 5790, Bloco 32, sala 07 - 87020-900 - Maringá, PR - cabandrade@uem.br
} 
Segundo Garza et al. (2001), características morfológicas e fisiológicas, como a altura ou a reação a pragas, podem ser modificadas através da atuação de hormônios vegetais, naturais ou sintéticos. Atualmente têm sido muito utilizados os fitorreguladores não hormonais (ou sintéticos), como o clormequat, o paclobutrazol, o uniconazole entre outros.

O paclobutrazol tem sido bastante estudado, tanto na cultura do girassol como em outras culturas, por ser um regulador de crescimento que atua reduzindo o alongamento da haste (GIANFAGNA, 1988). Entretanto, a melhor época para sua aplicação, visando a maior redução em altura das plantas de girassol, ainda não está bem definida. Pallez et al. (2002) obtiveram bons resultados com a aplicação aos 14 dias após a emergência das plantas; já Almeida \& Pereira (1996) obtiveram uma maior redução das hastes de girassol aplicando paclobutrazol em plantas com 10 dias de idade. Quanto à forma de aplicação, Dasoju et al. (1998), observaram que esse produto é ativo quando aplicado no substrato de crescimento, mas tem pouca eficácia como retardante químico quando aplicado como spray foliar, por não ser translocado. Essas observações concordam com os resultados obtidos por Almeida \& Pereira (1996), que relataram que o produto aplicado ao solo de vasos causou nanismo, mas não teve efeito no crescimento da haste quando aplicado no ápice da planta.

Para que se possa obter plantas totalmente isentas de contaminações, o uso de sistemas hidropônicos tem aumentado e, de acordo com Bliska Júnior \& Honório (1996) e Furlani et al. (1999), diversos sistemas podem ser usados, entre eles aquele com substratos, onde são utilizados vasos ou canaletas com material inerte, onde se aplica a solução nutritiva.

Quanto à composição da solução nutritiva, Sarruge (1975), ressaltava que não havia uma solução superior a outras. Diversas soluções vêm sendo empregadas, como as de Hoagland \& Arnon, Johnson e Hoagland modificada por Epstein \& Bloom (2004), etc. Entretanto, nas tabelas que citam as concentrações de nutrientes recomendadas para o cultivo hidropônico, não há referência à cultura do girassol. Essa ausência pode ser explicada pelo fato das pesquisas com girassol, como planta ornamental, serem bastante recentes.

Assim,objetivou-se avaliar o crescimento e produção de flores do girassol cultivado em hidroponia, no sistema com substratos, com solução nutritiva contendo diferentes dosagens do paclobutrazol.

\section{MATERIAL E MÉTODOS}

O experimento foi realizado no período de dezembro de 2005 a abril de 2006, em casa de vegetação, na Embrapa Soja, na cidade de Londrina, Paraná.
O cultivo das plantas foi realizado num sistema hidropônico, utilizando-se vasos com substrato inerte de sílica, onde se aplicou a solução nutritiva e aeração permanente através de compressor de ar. Foram utilizados vasos brancos, com capacidade para 3,6 litros, contendo 4,3 kg de sílica moída e 1,4 L da solução nutritiva de Hoagland, modificada por Epstein \& Bloom (2004).

Os tratamentos foram dispostos num esquema fatorial de 2x6, em um delineamento experimental de blocos casualizados, com dois (2) genótipos, seis (6) tratamentos e cinco (5) repetições, totalizando 60 parcelas. Os genótipos BRS Oásis e Helio 358 foram submetidos às doses $0 ; 0,5 ; 1$; 2; 4 e 6 mg. $\mathrm{L}^{-1}$ de paclobutrazol.

As sementes foram germinadas em bandejas de isopor preenchidas com areia, e umedecidas somente com água destilada. Quatro dias após a emergência, as mudas foram transplantadas para os vasos com sílica, mantendo-se uma planta por vaso. As plantas permaneceram em solução nutritiva diluída a $25 \%$ e $50 \%$, respectivamente do $4^{\circ}$ ao $6^{\circ}$ e do $7^{\circ}$ ao $9^{\circ}$ dia, após o transplante. Dez dias após o transplante, no final da tarde, iniciaram-se os tratamentos pela aplicação de paclobutrazol diretamente nos vasos, através de uma pipeta volumétrica nas doses previamente definidas, e pela substituição da solução nutritiva diluída por uma solução completa. Os vasos receberam complementação de água diariamente, mantendo-se o volume constante, e a cada 15 dias promoveu-se a troca total da solução nutritiva.

O desenvolvimento vegetativo foi avaliado pela altura da planta a cada dois dias (medidos com trena, a partir da inserção dos cotilédones até a última folha emitida), bem como pela altura final (medida também com trena, a partir da inserção dos cotilédones até a parte inferior do capítulo) e o diâmetro dos capítulos (medido com paquímetro), na fase de floração (R5). Os resultados foram submetidos à análise de variação (Teste F), utilizando-se as rotinas do SAS/STAT, aplicando-se para os valores significativos a $5 \%$ de probabilidade, a regressão linear e não linear para a análise dos parâmetros aferidos, em função das doses do regulador de crescimento.

\section{RESULTADOS E DISCUSSÃO}

\section{Altura das plantas por genótipo}

Observando-se as figuras 1 e 2, que relacionam as doses do regulador de crescimento com o tempo, verificamse diferenças de comportamento entre os genótipos.

Para o genótipo BRS Oásis, as plantas-controle tiveram uma curva ascendente mais acentuada, com aumentos crescentes a partir de $2 \mathrm{~cm}$, verificados do $11^{\circ}$ ao 
$14^{\circ}$ dia após a emergência (DAE), para valor máximo de 20 $\mathrm{cm}$, entre o $40^{\circ}$ e o $43^{\circ}$ DAE. Para os tratamentos 0,5 e $1 \mathrm{mg} . \mathrm{L}^{-1}$ não se pode diferenciar os ciclos de crescimento, embora tenha sido verificado o efeito inibidor do regulador até o $28^{\circ}$ DAE,em relação ao controle.

Entretanto, para os tratamentos de 2 e $4 \mathrm{mg} . \mathrm{L}^{-1}$, verificou-se 3 fases de desenvolvimento das plantas, sendo a primeira da emergência até os 38 DAE, com crescimento médio de 3 e $2 \mathrm{~cm}$, respectivamente, a cada
3 dias; a segunda fase, dos 38 aos 60 dias, foi a de maior crescimento, atingindo $9 \mathrm{~cm}$ a cada 3 dias, e finalmente a terceira, que foi de estabilização, durante a emissão do botão floral, com crescimento de 2 e $1 \mathrm{~cm}$ a cada 3 dias.

O tratamento de $6 \mathrm{mg} . \mathrm{L}^{-1}$ mostrou plantas com crescimento reduzido e estáveis durante todo o ciclo, com elongação máxima de $4 \mathrm{~cm}$ a cada 3 dias, com altura final de aproximadamente $35 \mathrm{~cm}$.

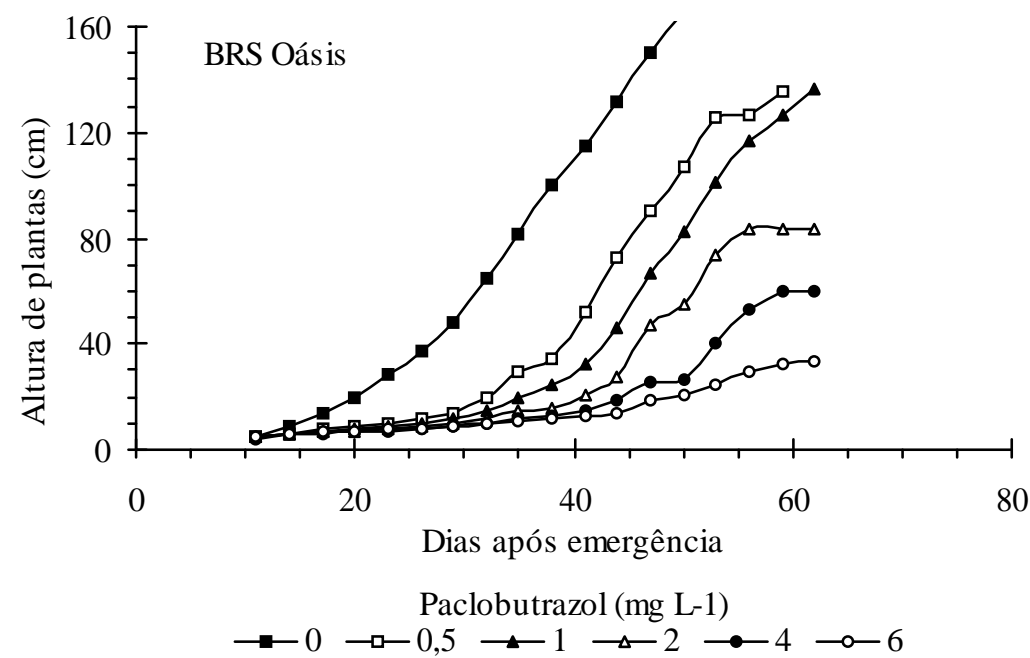

Figura 1 -Altura de plantas $(\mathrm{cm})$ do genótipo BRS Oásis em função do tempo e do efeito das doses de paclobutrazol $\left(0 ; 0,5 ; 1 ; 2 ; 4\right.$ e 6 mg.. - $\left.^{-1}\right)$.

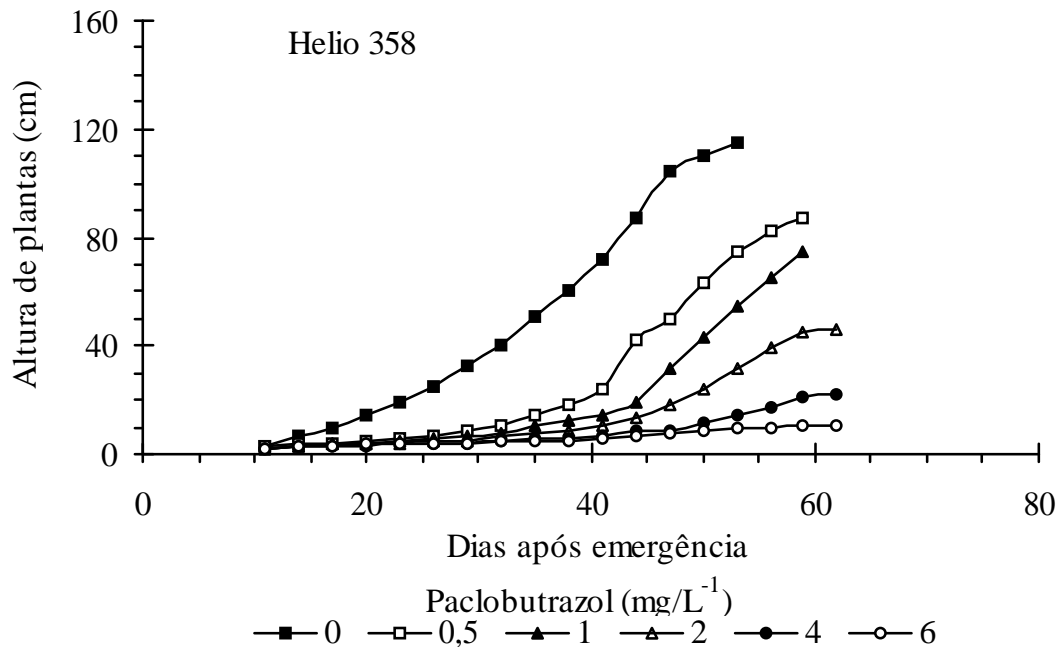

Figura 2 - Altura de plantas $(\mathrm{cm})$ do genótipo Helio 358 em função do tempo e do efeito das doses de paclobutrazol $\left(0 ; 0,5 ; 1 ; 2 ; 4\right.$ e 6 mg. $\left.\mathrm{L}^{-1}\right)$. 
As plantas-controle do genótipo Helio 358 apresentaram uma curva de crescimento, dividida em 3 fases: a primeira, que foi da emergência até os $26 \mathrm{DAE}$, onde as plantas apresentam um crescimento normal (em média $4 \mathrm{~cm}$ a cada 3 dias); a segunda fase foi a de maior crescimento, estendendo-se do $26^{\circ}$ até o $41^{\circ} \mathrm{DAE}$, onde as plantas cresceram $10 \mathrm{~cm}$ a cada 3 dias; e o terceiro ciclo, a partir do $41^{\circ}$ dia até a colheita, com aproximadamente 50 dias, com crescimento de $7 \mathrm{~cm}$ a cada 3 dias.

Essa mesma tendência de crescimento em fases foi observada nas plantas dos tratamentos 0,$5 ; 1$ e 2 mg.L . $^{-1}$. Entretanto, para o tratamento de $2 \mathrm{mg} . \mathrm{L}^{-1}$, o que se observou foi que a segunda fase, que representaria a fase de maior crescimento, iniciou-se mais tardiamente - no $41^{\circ}$ dia, e teve menor duração - apenas 9 dias, quando se estabilizou para a emissão do botão floral, o que resultou em uma altura final de cerca de $42 \mathrm{~cm}$.

Já para os tratamentos de 4 e $6 \mathrm{mg} . \mathrm{L}^{-1}$, não se notou essa divisão do crescimento em fases, constatando-se que o efeito do regulador de crescimento manteve-se durante todo o desenvolvimento da planta, que alcançaram somente 25 e $15 \mathrm{~cm}$ de altura, respectivamente.

Observou-se efeito do paclobutrazol até os 35 dias de idade, em média, para ambos os genótipos, com plantas apresentando internódios mais curtos. Após esse período, esgotou-se o efeito do regulador de crescimento e os internódios apresentaram-se maiores.

Verificou-se que o interessante é reduzir o período que compreende a segunda fase, mas possibilitando uma curva de crescimento semelhante à normal, pois esse crescimento pode estar associado à definição do tamanho do capítulo, visto que a planta nessa segunda fase tem seu maior crescimento vegetativo, ou seja, reduzindo essa fase, haverá redução na altura final da planta sem, entretanto, que isso altere substancialmente a formação do capítulo, que ocorre na fase seguinte, no processo reprodutivo, que é a fase de estabilização de altura.

\section{Altura final das plantas}

A aplicação de paclobutrazol proporcionou redução do crescimento tanto para BRS Oásis quanto para Helio 358. As taxas de redução correlacionaram-se com os níveis do regulador de crescimento segundo um modelo exponencial, porém os efeitos foram mais intensos sobre o genótipo Helio 358. Ele apresentou, mesmo na ausência do tratamento, menor altura final, demonstrando haver influência genética sobre os resultados, fato também constatado por Pallez et al. (2002) e Whipker \& McCall (2000). Segundo Dasoju et al. (1998), plantas de girassol mostraram maior redução da altura conforme aumento da dose de paclobutrazol, fato que também foi constatado neste trabalho. Incrocci et al. (2003), concluíram que o tratamento com o regulador de crescimento não somente reduziu a altura das plantas, como também aumentou a uniformidade das mesmas. Esse fato corrobora as informações de Vernieri et al. (2003), que atestaram que, além de ter ocorrido aumento na uniformidade das plantas, não houve efeito negativo na qualidade das mesmas.

A aplicação de paclobutrazol na dose de $0,5 \mathrm{mg} . \mathrm{L}^{-1}$, reduziu cerca de $18 \%$ a altura final das plantas para os dois genótipos estudados. Para a dose de $2 \mathrm{mg} . \mathrm{L}^{-1}$, a redução foi de $41 \%$ para BRS Oásis e de $54 \%$ para Helio 358, quando comparadas ao controle. Para a dose de 6 mg.L. $\mathrm{L}^{-1}$, a redução foi de $79 \%$ para BRS Oásis e chegou a 91\% para Helio 358 (Figura 3).

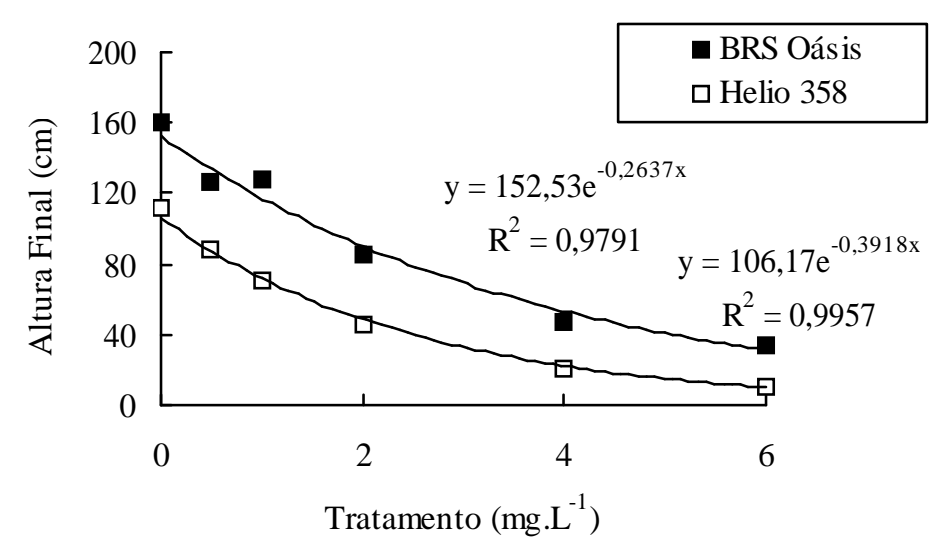

Figura 3 - Altura final de plantas $(\mathrm{cm})$ de girassol dos genótipos BRS Oásis e Helio 358 em função dos tratamentos com paclobutrazol $(0 ; 0,5 ; 1 ; 2 ; 4$ e 6 mg.L-1 $)$. 


\section{Diâmetro de Capítulo}

Para ambos os genótipos, houve redução do diâmetro de capítulo com o aumento das doses de paclobutrazol, de modo semelhante. Na dose de $0,5 \mathrm{mg} . \mathrm{L}^{-1}$, a redução no diâmetro dos capítulos foi de $10 \%$ em relação ao controle, de $30 \%$ na dose de 2 mg. $L^{-1}$ e chegou a $70 \%$ na dose de 6 $\mathrm{mg} . \mathrm{L}^{-1}$. Esse efeito não é desejado e limita a utilização de doses elevadas do produto, apesar dos efeitos positivos sobre a altura de plantas (figura 4).

Resultados diferentes foram encontrados por Dasoju et al. (1998) e Whipker \& McCall (2000), os quais relataram que, embora o diâmetro de capítulo tenha sido estatisticamente menor, as diferenças não foram prejudiciais ao aspecto comercial. Também Pallez et al. (2002) concluíram em seu trabalho que o paclobutrazol diminuiu o diâmetro do capítulo, entretanto, esse decréscimo não teve importância comercial. Já Whipker \& Dasoju (1998) relataram que a aplicação de paclobutrazol não afetou o diâmetro de capítulo; isso ocorreu provavelmente porque eles utilizaram o produto somente em aplicação foliar e não diretamente no substrato. Segundo Anefalos \& Guilhoto (2003) e Dasoju et al. (1998), o girassol tem um grande potencial como planta ornamental, pelo tempo curto para sua colheita, facilidade de propagação e principalmente pela sua inflorescência muito atrativa e bastante procurada para ornamentação em vasos e confecção de arranjos florais. Para ornamentação em vasos, o tamanho do capítulo assume uma maior importância, pois ele deve ser proporcional ao tamanho do vaso onde será produzido e comercializado. Por isso é que uma redução na altura final da haste não poderá reduzir drasticamente o tamanho do capítulo, pois do contrário, irá perder o valor comercial.

Quanto ao sistema hidropônico que foi utilizado, Martinez (1996) cita que, na Europa e Estados Unidos, o cultivo hidropônico tem produzido flores de melhor aspecto fitossanitário e nutricional, aumentando a vida em pós colheita das flores cortadas. Rodrigues (2002) ressalta a facilidade de se trabalhar com hidroponia com grânulos inertes, como a sílica, pois os redutores de crescimento afetam somente a altura final das plantas e o tamanho do capítulo, mas não reagem com o substrato de crescimento.

As médias das variáveis analisadas e os quadrados médios obtidos apresentam-se nos Quadros 1 e 2.

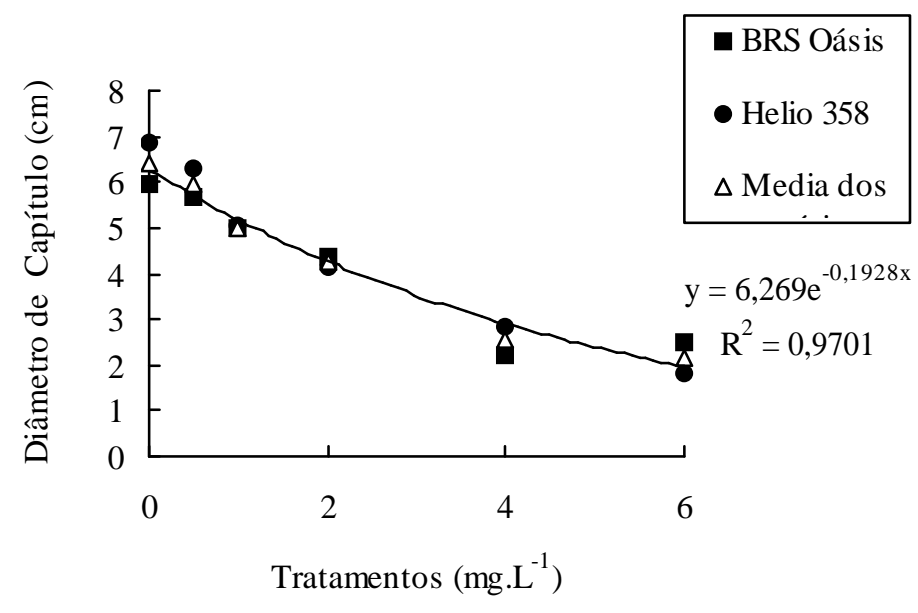

Figura 4 - Diâmetro de capítulo (cm) em função dos tratamentos com paclobutrazol $(0 ; 0,5 ; 1 ; 2 ; 4$ e 6 mg.L-1 $)$. 
Quadro 1 - Médias das variáveis avaliadas, Diâmetro de Capítulo (DiâmCap) e Altura Final (AltFinal) para os genótipos e as doses de paclobutrazol.

\begin{tabular}{|lllc|}
\hline Genótipo & ${\text { Dose } \mathrm{mgL}^{-1}}^{-1}$ & Diâm Cap $(\mathrm{cm})$ & Alt Final $(\mathrm{cm})$ \\
\hline BRSOásis & 0 & 5,98 & 160,46 \\
& 0,5 & 5,68 & 126,22 \\
& 1,0 & 5,00 & 128,22 \\
& 2,0 & 4,38 & 85,94 \\
& 4,0 & 2,20 & 46,84 \\
& 6,0 & 2,50 & 34,28 \\
\hline Helio 358 & 0 & 6,86 & 112,44 \\
& 0,5 & 6,28 & 88,10 \\
& 1,0 & 5,04 & 71,26 \\
& 2,0 & 4,17 & 46,17 \\
& 4,0 & 2,86 & 20,38 \\
& 6,0 & 1,82 & 10,87 \\
\hline CV $(\%)$ & & 16,0 & 9,8 \\
\hline
\end{tabular}

Quadro 2 - Quadrados médios obtidos na análise de variância para os parâmetros Diâmetro de Capítulo (Diâm.Cap) e Altura Final (Alt.Final). Londrina, Paraná, 2006.

\begin{tabular}{|lrcc|}
\hline & \multicolumn{3}{c|}{ Quadrado Médio } \\
\hline Fontes de Variação & G. L. & Diâm.Cap. & Alt. Final \\
Blocos & 4 & 0,79 & 209,6 \\
Genótipos & 1 & 0,65 & $26200,1^{* * *}$ \\
Doses & 5 & $28,8 * * *$ & $18579,4^{* * *}$ \\
Interação Genótipo x Doses & 5 & 0,81 & $553,1^{* * *}$ \\
\hline
\end{tabular}

* Significativo a $5 \%$ de probabilidade

** Significativo a $1 \%$ de probabilidade

\section{CONCLUSÕES}

Existem diferenças genéticas na resposta ao paclobutrazol aplicado no substrato de crescimento. O híbrido comercial Helio 358 revelou-se mais adequado para a produção de girassol como planta ornamental, com doses entre 1 e $2 \mathrm{mgL}^{-1}$ de paclobutrazol, pois apresentou redução de capítulo proporcional à redução de altura das plantas.

A dose mais adequada para manutenção do tamanho do capítulo foi $0,5 \mathrm{mgL}^{-1}$, enquanto a exigida para redução de altura final das plantas foi de $2 \mathrm{mgL}^{-1}$. Entretanto, nas doses maiores houve redução na altura final das plantas, mas os pecíolos continuaram longos, os capítulos foram reduzidos e perderam o valor comercial, as raízes e os caules ficaram menores e mais finos.
O sistema hidropônico, em substrato com o uso de sílica, mostrou-se viável para a produção de girassol ornamental.

\section{REFERÊNCIAS BIBLIOGRÁFICAS}

ALMEIDA, J. A. S. de; PEREIRA, M. de F. D. A. Efeito de GA3 e paclobutrazol no desenvolvimento vegetativo do girassol. Revista Brasileira de Fisiologia Vegetal, Londrina, v. 9, n. 1, p. 53-58, 1996.

ANEFALOS, L. C.; GUILHOTO, J. J. M. Estrutura do mercado brasileiro de flores e plantas ornamentais. Agricultura em São Paulo, São Paulo, v. 50, n. 2, p. 41-63, 2003. 
BLISKA JÚNIOR, A.; HONORIO, S. L. Cartilha tecnológica: hidroponia. [S.1.]: Faculdade de Engenharia Agrícola, 1996.

DALL'AGNOL, A.; VIEIRA, O. V.; LEITE, M. R. V. B. de C. Origem e histórico do Girassol. In: Girassol no

Brasil. Londrina: Embrapa Soja, 2005. v. 1, p. 1-12.

DASOJU, S.; EVANS, M. R.; WHIPKER, B. E. Paclobutrazol drenches control growth of potted sunflowers. HortTechnology, Alexandria, v. 8, n. 2, p. 235-237, 1998.

EPSTEIN, E.; BLOOM, A. J. Mineral nutrition of plants: principles and perspectives. Sunderland: Sinauer Associates, 2004. 400 p.

FURLANI, P. R.; SILVEIRA, L. C. P.; BOLONHEZI, D.; FAQUIN, V. Cultivo hidropônico de plantas. Campinas: IAC, 1999. 52 p. (Boletim técnico, 180).

GARZA, M. S.; GONZALEZ, H. G.; GARCIA, F. Z.; HERNANDEZ, B. C.; GARCIDUENAS, M. R. Efecto de cuatro fitoreguladores comerciales en Desarrollo y rendimiento del girasol. Ciencia Vanl, [S.1.], v. 4, n. 1, 2001.

GIANFAGNA, T. J. Natural and synthetic growth regulators and their use in horticultural and agronomic crops. In: Plant hormones and their role in plant growth and development. Davies: Kluwer Academia, 1988. 681 p.

INCROCCI, G.; MUGNAI, S.; VERNIERI, P.; SERRA, G.; TOGNONI, F. La produzione del Girasole da vaso fiorito. Colture Protette, [S.1.], v. 32, n. 2, p. 105-114, 2003.

MARTINEZ, H. A hidroponia das flores: parte II. Boletim Hidropomanias \& Cia, [S.l.], jul. 1996.
PALLEZ, L. C.; DOLE, J. M.; WHIPKER, B. E. Production and post production studies with potted sunflowers. HortTechnology, Alexandria, v. 12, n. 2, p. 206-210, Apr./ June 2002.

REYES, F. G. R.; GARIBAY, C. B.; UNGARO, M. R. G.; TOLEDO, M. C. F. Girassol: cultura e aspectos químicos, nutricionais e tecnológicos. Campinas: Fundação Cargill, 1985. $86 \mathrm{p}$.

RODRIGUES, L. R. F. Técnicas de cultivo hidropônico e de controle ambiental no manejo de pragas, doenças e nutrição vegetal em ambiente protegido. Jaboticabal: FUNEP/UNESP, 2002. 762 p.

SARRUGE, J. R. Soluções nutritivas: nota técnica. Summa Phytopathologica, Jaguariúna, v. 1, p. 231-233, 1975.

VERNIERI, P.; INCROCCI, G.; TOGNONI, F.; SERRA, G. Effect of cultivar, timing, growth retardants, potting type on potted sunflowers production. In: INTERNATIONAL SYMPOSIUM ON PROTECTED CULTIVATION IN MILD WINTER CLIMATE: PRODUCT AND PROCESS INNOVATION, 6., 2003, The Hague. Proceedings... The Hague: [s.n.], 2003.

WHIPKER, B. E.; MCCALL, I. Response of potted sunflower cultivar to daminozide foliar sprays and paclobutrazol drenches. HortTechnology, Alexandria, v. 10, n. 1, p. 209-211, 2000.

WHIPKER, B. E.; DASOJU, S. Potted sunflower growth and flowering responses to foliar applications of daminozide, paclobutrazol and uniconazole. HortTechnology, Alexandria, v. 8, n. 1, p. 86-88, Jan./Mar. 1998. 